\title{
Greater Occipital Nerve Block: a minimally invasive alternative to blood patch for Post Dural Puncture Headache treatment.
}

\section{J.Stephan, H.Saba, A.Azzi, K.Maroun, T.Samir, A.Neemtallah, G.Anthony, S.dalia,}

\section{S.Chamandi.}

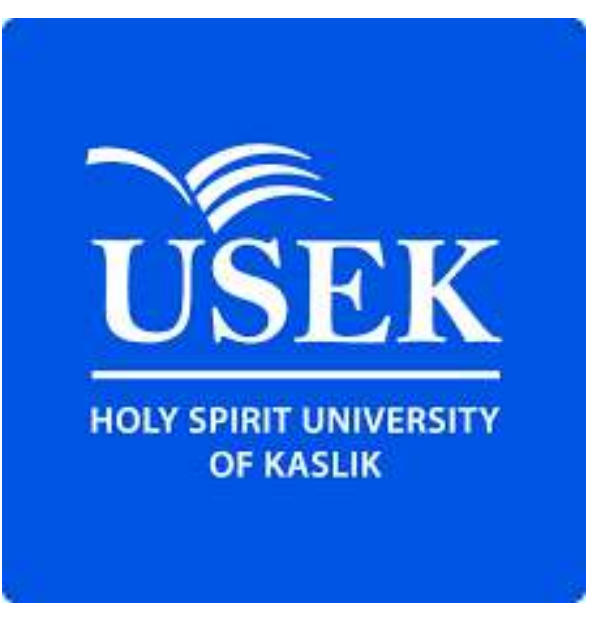

\section{Background and Aim}

Post Dural Puncture Headache (PDPH) is a common complication after inadvertent dural puncture. It is described as severe postural headache that appears within 72 hours, is aggravated by standing position, and relieved by lying down. Treatment is conservative within the first 48 hours, it includes bed rest, IV hydration, caffeine and analgesics administration. If symptoms persist, invasive procedures such as saline or blood patch should be considered. In our preliminary study we attempted to perform an occipital nerve bloc after failure of conservative treatment as an alternative to blood patch.

\section{Methods:}

11 female patients diagnosed with PDPH were included in the study after failure of conservative treatment. Ultrasound guided Greater Occipital Nerve Block (GONB) was performed using $5 \mathrm{ml}$ of bupivacaine and dexamethasone. When there was no improvement after GONB, a blood patch was performed.

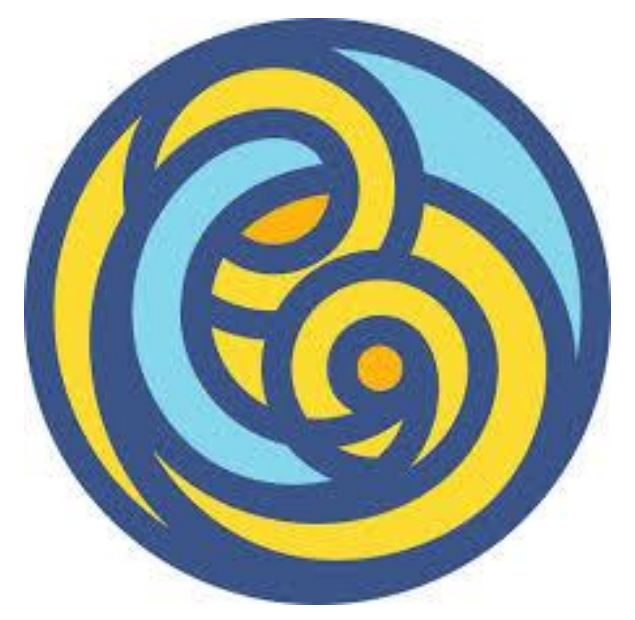

NDS-UH

Results:

6 Patients showed complete improvement of symptoms after GONB, while 5 patients underwent a blood patch 3 days post GONB.

\section{Conclusion:}

Our preliminary data suggests that GONB might reduce the need for blood patch. It is considered as a minimally invasive procedure, and effective in treating PDPH that is unresponsive to conservative treatment.

\section{Key words}

Post dural puncture headache, blood patch, occipital nerve block, postural headache.

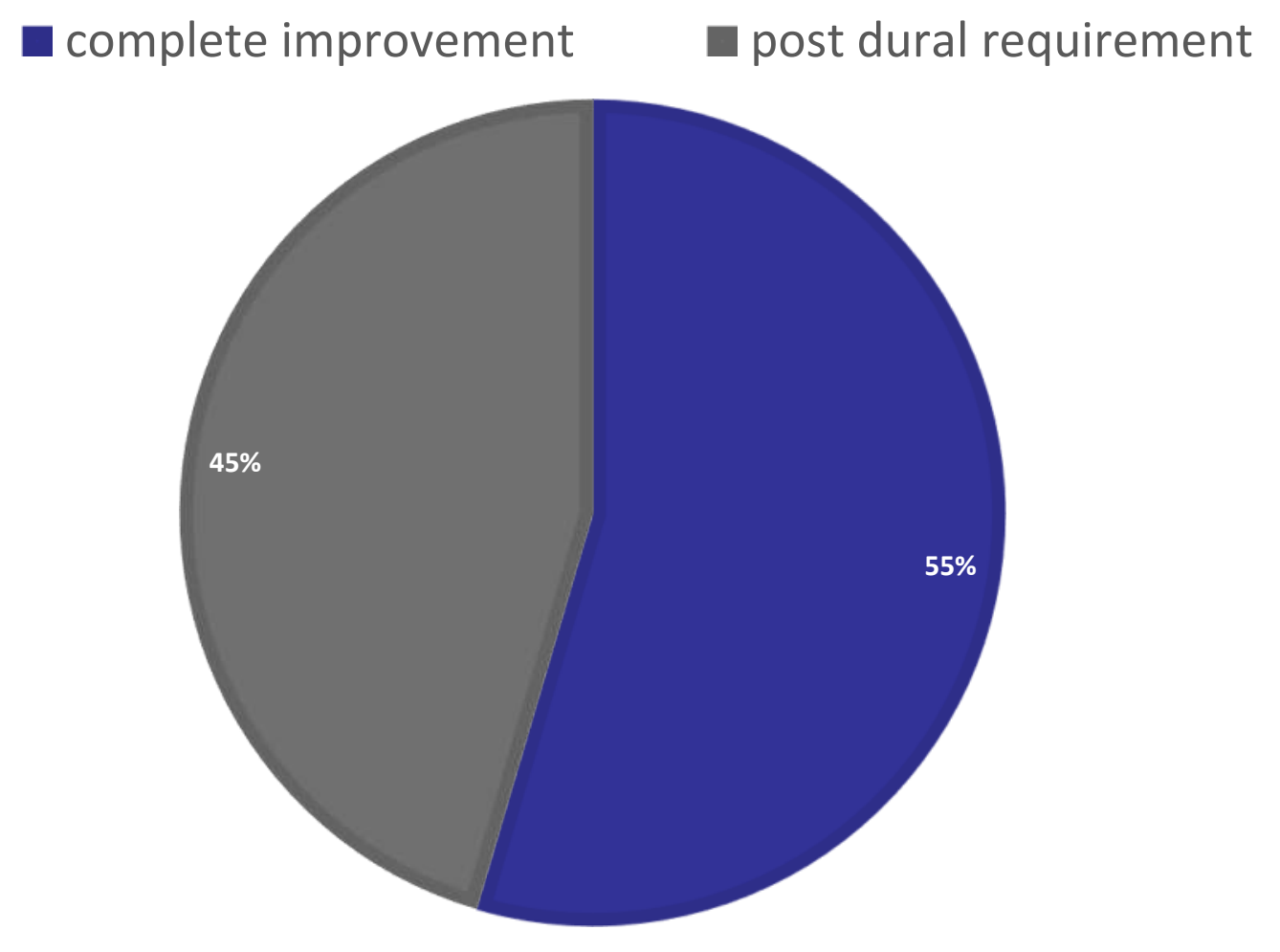

